\title{
Cross Layered Design and RF Energy Harvesting Techniques: A Survey
}

\author{
${ }^{*}$ Mr. Shiva Kumar ${ }^{1}$, Dr. Rajashree V. Biradar ${ }^{2}$ \\ Assistant Professor, Dept. of CSE,RYMEC, Ballari, Karnataka, India \\ Professor, Dept. of CSE, BITM, Ballari,Karnataka, India
}

\begin{abstract}
In recent years, energy optimization is one of the the major concern for research community while designing an Self Sustainable Energy Efficient Wireless Sensor Networks. In this paper, we review the different cross-layer architectures based on cross layer design and the available RF energy-harvesting mechanisms. In the cross-layer design, different parameters are exchanged between the adjacent layers to ensure efficient use of energy. Energy scavenging is the process of harvesting energy from the ambient sources (e.g., solar power, RF energy ,thermal energy, wind energy, salinity gradients), stored in storage devices like batteries, and is used to power up small wireless electronic devices and wireless sensor networks.
\end{abstract}

Keywords: WSN, energy harvesting, cross layered design.

\section{INTRODUCTION}

Wireless sensor networks are geographically grouped discrete and dedicated sensors for observing and maintaining a record of the environmental conditions and restructuring the collected data at a centralized location. WSNs are used to calibrate weather conditions like temperature, sound, contamination levels, dampness, wind, and so on.

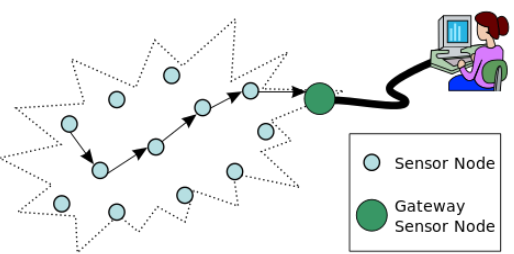

Fig. 1.Wireless Sensor Network

Wireless sensor networks are made up of small nodes that are capable of sensing, computing, and wireless networking, representing a collection of three important technologies. In most of the scenarios, nodes depend on the energy supply using batteries. Replacing these energy sources very often is usually not feasible. WSN must be operative until the mission completes or to a best possible extent of time. Therefore, the lifetime of a sensor node becomes as one of the prime figure of merit. Thus, energy requirements for sensor nodes can only be satisfied through cross-layer designs.

The cross-layer design shares the vital information among the layers for the better performance, which would violate the TCP/IP model. A lot of research has been presented to achieve the cross-layer designs in wireless networks. Many of the cross-layer designs are proposed using appropriate mechanisms in the lower layer of TCP/IP [1], [2].

One of the prime objective of the Cross layered design is to achieve an optimal energy solution by information extraction and usage from the non adjacent layers of TCP/IP.The concept of cross-layering was first proposed for TCP/IP networks, when wireless links were deployed [3],[4]. Since the proposed TCP/IP stack was for wired connections, there was a decline in the performance of wireless technology when they were considered as part of it. Lately, the concept of cross-layering has drawn more attention in WSNs research and it is still in its early development [4]. However, considerable solutions are presented in the literature, numerical frameworks and simulations, they claim to have attained improved performance gains than that of the available schemes. Prime goals of cross-layer optimizations in WSNs are reduction of energy consumption [5], efficient routing [6], QoS provisioning [7], and optimal scheduling [8].Some of the new protocols implemented on cross- layer design reveal the optimizations they consider in order to have a small database on what has been proposed so far.

Energy is the prime resource to maintain the operations of any Wireless device. The life span of wireless devices is limited because of the limited storage capacity of batteries . Harvesting energy from the surroundings of the existing sources has become one of the promising technologies available. There are number of ambient energy resources like sunlight, temperature responses, mechanical vibrations, radio frequency (RF) and EM radiations in the environment [9] etc,. Power density is the main parameter to harvest energy from any of the existing energy resources, and the sunlight has the maximum density [10]. During day the normal power density is recorded as $100 \mathrm{~mW} / \mathrm{cm} 2$ [11] and it comes down to zero in the absence of sunlight. And moreover the solar panels require larger area for implementation. In the spectrum of electromagnetic waves RF has a wide frequency band of 3 $\mathrm{kHz}$ to $300 \mathrm{GHz}$ that are radiated from millions of radio stations around the globe. The fundamentals of electromagnetic wave transmission were first offered by Tesla [12] in 1899. In the available energy resources, radio frequency (RF) energy has the smallest power density ranging $0.2 \mathrm{nW} / \mathrm{cm} 2$ to $1 \mu \mathrm{W} / \mathrm{cm} 2[13-14]$. On the other hand, Radio Frequency energy can be found in the surrounding ambient RF sources including MF (AM Radio, 526.5-1705 KHz); FM (87.5-108 MHz); TV (41- 
$250 \mathrm{MHz}, \quad 470-950 \mathrm{MHz}) ; \quad$ GSM $\quad(850 / 1900$ or 900/1800 MHz); CDMA, 3G, 4G, and ISM (industrial scientific medical, $2400 \mathrm{MHz})$; and Wi-Fi (2.45/5.0 GHz). [15-17].

Moreover, the RF energy is least affected by varying climatic conditions. These advantages make the broadcasting stations as the most favorable energy sources to wireless sensor nodes for SHM applications.[18]. The RF energy harvesting may be one of the prime source of energy supply for many wireless application of wireless sensor networks (WSNs) and internet of things. The wireless sensor networks find their use in military applications such as battlefield surveillance, industry applications ,consumer applications ,Health monitoring and so on.

As shown in the Fig.2, an RF energy scavenging circuit made-up of an dipole antenna, a impedance matching circuit, a rectifier, a voltage multiplier, and an energy storage device or load, as discussed in the following sections. Normally in an Radio Frequency energy Scavenging/Harvesting system, the radiated RF signals communicating through wireless medium are tapped by a single or more than one RF antennas. The maximum power transfer is provided by using asuitable matching circuit. Since the output voltage of the rectifier is normally very low, multiplier is used to boost the DC voltage level to drive a wireless device. Finally energy is preserved in batteries or sometimes directly connected to drive the devices. In this paper, we also try to point out the parameters used to assess the throughput of an RF power scavenging mechanism, by using the power conversion efficiency (PCE) of the system.

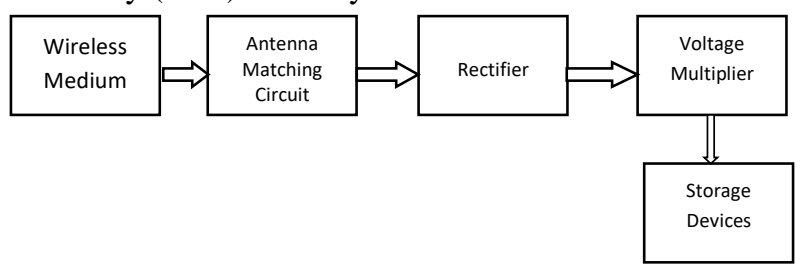

Fig. 2. RadioFrequency Energy Harvesting System

The remaining section of this paper is arranged as follows. Second Section addresses the problems frequently faced by cross layer approaches and RF energy Harvesting schemes. In the third Section various components and techniques of energy harvesting are discussed. Finally, in the fourth Section the conclusions are made.

\section{- Propagation Model:-}

The propagation model is one of the vital parameter in the design and implementation of a wireless sensor network (WSN).It is a collection of mathematical expressions that describes the radio wave propagation characteristics such as operating frequency, distance of the source from the receiver, signal strength and other conditions. Normally one model is developed and is used to predict the performance of all other propagation models with similar limitations. The formulated models predict the path loss and effective coverage of transmitted waves which is calculated theoretically or practically.It is practically impossible to formulate the exact path loss and other parameters using a single mathematical formulation since the RF signals are propagated under different environmental and geographical conditions. Therefore various attenuation models exists based upon the different operating conditions such as indoor, outdoor and free space. One of the widely used and optimistic model is the free-space model [87], known as Friis transmission formula, which emphasizes that under ideal conditions , the power around the antenna is equally distributed. The free space path loss (FSPL) is measured as given in Equation.1.

$$
\text { FSPL }=\frac{P_{L}}{P_{T}}=\left(\frac{4 \pi d}{\lambda}\right)^{2}(1)
$$

Where, $\mathrm{P}_{\mathrm{t}}$ is the transmitter power, $\mathrm{P}_{\mathrm{r}}$ is the receiver power, $\mathrm{d}$ is the distance between the transmitter and receiver, $\lambda$ is the wavelength.

\section{DIFFERENT CROSS LAYER APPROACHES BASED ON PROTOCOLS \\ 2.1 Routing Protocol}

Ammar et al., (2018), Proposed a scheme of cross-layer design that includes physical and network layer which significantly extends the network lifetime enhancing the communication efficiency level. Results claim an improvement in ad hoc on-demand distance vector protocol that is used to discover the route and provide reliable transmission of data by considering the two energy thresholds of SNR and the residual energy along with the power minimizing algorithm.

Chen et al., (2010) Proposed a communication scheme that reduces the intra communication in virtual MIMO nodes, saving the energy and minimizing the latency concurrently at transmission links. The proposed routing protocol claims to optimise energy and latency along the route.

Di Marco et al., (2010) Propose TREnD algorithm which is a simple cross-layer protocol to control applications over WSNs. It claims to satisfy application conditions on reliability and latency while reducing the energy consumption. TREnD is allows the network to accommodate itself for changing requirements. The parameters considered are the sleeping discipline and beacon mechanism, that enhances reliability and energy efficiency.

Babulal et al., (2010) Proposed an XLE2R (Cross layer Energy Efficient Routing) algorithm based on routing decision to prolong network lifetime for static nodes. It depends on the communication among various layers and the information of source and the destination node so as to increase the throughput of sensors. Comparison of DSR with XLE2R are also shown.

Chen et al., (2010) Propose a design to reduce the total power consumed by multiple-hop wireless sensor networks for providing target end-to-end transmission and reliability. By using a cross-layer design various methods are presented for direct transmission, single and multirelay cooperation scenario .

Chabalala S. C. et al., (2011) Propose a cross-layer adaptive routing (CLAR) protocol for WSNs, that claims to reduce the application of routing control packets to increase network-lifetime. They have developed an 
analytical model to estimate energy consumed during route establishment and existing simulation results that claim better performance and energy efficiency.

Dessales et al., (2010) Propose a building monitoring systems that are used to identify and increase the building energy performances in an efficient way. They have emphasized the importance of cross-layer approach between the physical layer and the MAC layer.

Gao et al., (2013) Proposed a multiple-hopping routing protocol based on the LAR algorithm and cross-layer mechanism. Distance between the nodes and the equilibrium weight factors are the two parameters considered to minimize the energy consumed and limit the packet drop rate of the network.

Jaradat et al., (2013) Propose a cross layer design by implementing an algorithm that makes use of the parameters such as the left over battery power, link strength and the power required for transmission. The designed algorithm is an energy aware routing algorithm that improves the overall power consumed and maximizes the network lifetime by determining the subsequent hop to relay node so as to reach the network sink node.

Anupama et al., (2014) Propose a procedure for monitoring the pipeline system with the help of the Wireless Sensor Network technology by considering over 100 wireless motes to monitor gas and oil pipelines. The procedure is used to develop a protocol stack that consists of Time Synchronization, Topology Control, Routing, and MAC protocols. The stack of protocol reveals some important characteristics such as reduced energy consumed and memory utilization that play a major role in implementation of wireless sensor networks.

Chen et al., (2015) Proposed cross layered design implements routing protocol with improved energy efficiency and reliability. The scheme is based on to optimize the energy consumed and provide end-to end reliability for WSN. Results claim improved energy efficiency and reliability over the existing algorithms.

Alhalafi et al., (2016) Propose an "green Task-Based Sensing" (gTBS) scheme that uses energy efficient cross layer design. The scheme not only avoids the power wasted in unnecessary signaling but also improves reliability and efficiency of the network life time.The gTBS uses the sleep and wake-up scheme that allows the inactive nodes to sleep and reduce the power consumed .

Mishra et al., (2017) Proposed a C-L design that minimizes the power taken and improves network lifetime by involving adaptive grid-based data aggregation and routing algorithms for data transmission.

Shibo et al., (2012) Proposed JRPRA a distributed algorithm to improve network lifetime that jointly considers routing, power control, and link-layer random access.

\subsection{Medium Access Control layer protocols}

Zhang et al., (2010) Proposed an review over few parameters of WSN and introduced of some recent typical MAC protocols with respect to energy efficiency for WSN. The channels accessing policies discussed are grouped as contention-based, TDMA-based, hybrid, and cross layer protocols.
Dessales et al., (2010) Proposed a realistic propagation model that considers all the climatic conditions. The quality of radio link is evaluated by using the BER and estimate the power consumed to transmit every bit successfully. The power gain is achieved by considering the joint optimization of the Medium Access Control sub layer and the Physical link layer.

Shen et al., (2011) propose the comparison of the power efficiency of MAC protocols in WSNs. According to channel access policy the MAC protocols are grouped as contention-based, TDMA-based, hybrid, and cross layer MAC protocols, comparison is carried out by considering their advantages and disadvantages qualitatively. By implementing in ns-2 simulator the MAC protocols are reviewed in the view of energy efficiency by quantitative comparison.

Karvonen, et al., (2006) Propose a cross layer design that uses the forward error correction (FEC) coding and the identification of the sleep and wakeup periods for narrowband wireless sensor networks (WSNs). This scheme considers the information extracted from the lower two layers of TCP/IP.

Tiglao et al., (2012) Propose a NACK-based repair scheme that is combined with an adaptive MAC layer retransmission scheme to enhance the overall output of the protocols used in the transport layer. The delay incurred in end to end transmission is reduced while reliability and energy efficiency are maintained. The NACK algorithm automatically initiates intermediate retransmission of packet when an out-of-sequence packet is detected. Simulation results claim to improve throughput and energy efficiency.

Hsueh et al.,(2012) Propose a cross layer design that integrates MAC protocol at the receivers side.The protocol decreases the time taken for authentication and thereby reduce the power exhausting attacks. As a result of which the replay and forge attacks are addressed in an energy efficient way.

Sadouq et al.,(2014) Proposed cross layered design for energy-efficient framework for long-lasting sensor networks. The design comprises of physical layer, medium access control, and routing layer.The designed framework helps the node's energy to be used efficiently by reconfiguring and enabling them to redistribute the management tasks which in turn enhances the scalability of the overall system in heavily populated sensor networks. Sadiq et al., (2017) propose Energy-Efficient Cross-Layer (EECL) approach in which the network layer exploits the information of interaction between MAC and Physical layers to reduce the energy consumed in forwarding the data .The approach considers the X-MAC protocol that uses the distances between the nodes and BER as the parameters of interest.

Quintero et al., (2017) proposed a scheme that utilizes Bayesian inference and more accurately particle filtering to estimate the state of charge inside the voltage SOC curve. This statistics reveals valuable results that can be included in MAC protocols for energy efficient communication.

Cho et al.,(2007) Propose a cross-layer design termed as 
Cross Layer Medium Access Control (CLMAC) protocol to present energy-efficient routing. The CLMAC combines the information of network layer and link layer routing distance within a small routing table, This in turn helps the node to decrease the routing overhead and enhance the network life time of the sensor nodes.

Bencini, et al., (2010) Propose a cross-layer design that includes an MAC and Routing protocol integrated to manage defects encountered in Wireless Sensor Network (WSN). The designed protocol reveals some important parameters such as improved efficieny,reduced latency and reliable end to end transmission. The design automatically reconfigures the network topology on finding the defect without actually increasing the signaling overhead and the latency.

Song, et al., (2007) Propose a cross layer design that encompasses the Low Energy Self-Organizing Protocol (LESOP) for target tracking in heavily populated wireless sensor networks. The exchange of information between application layer and MAC is exploited .The protocol is further simplified by excluding Transport and Network layers. The LESOP is simple protocol that reduces the tradeoff among the tracking error and the network energy consumption. And also the LESOP becomes the first embedded wireless Interconnect architecture to be demonstrated as a two layer architecture .

Chen et al., (2015). Propose a cross layer design to decrease the power consumed in multi-hop wireless sensor networks while maintaining end to end reliable transmission. The design proposes optimization scheme for direct transmission, joint routing scheme for single-relay cooperation and power allocation scheme for multi-relay cooperation scenarios. The results presented reveal that the design consumes less power and is more reliable than the available algorithms.

\subsection{Communication protocol}

Xueying Zhangetal.,(2015) propose a TopologicalStructure Layered Configurations (TSLC) routing algorithm to improve the quality of WSN data transmission performance based on the topological structural characteristics and optimization of network layers. Using cross-layer design the co processing of layers is achieved by extracting the information of the nodes in the network and MAC layer. And the results reveal to save the power consumption of the entire network and enhance the performance of the network ,also prolong network life time.

\subsection{Transport layer protocol}

Xiong et al.,(2010) Propose a scheduling algorithm that uses the cross layer design concept in the process of decision making and thereby making the protocol channel adaptive. The algorithm is based on a Markov Decision Process (MDP) method that uses the present channel state and calculates the average energy cost incurred in the process of packet delivery is practiced. Numerical examples reveal the supremacy over the existing schemes.

\section{COMPONENTS AND TECHNIQUES OF ENERGY HARVESTING}

\subsection{RectEnna}

Rectenna is a special type of rectifying antenna that is used to convert the induced AC by the electromagnetic energy to DC. Usually the rectenna is made up of a simple dipole antenna with a RF diode connected across it. Normally the schottky diodes are preferred because of their high speed of switching.

Cao et al., (2017) Proposed an RF energy harvesting scheme that consists of twin coil ferrite rod (TCFR) antenna in amplitude modulation (AM) band. They claim $20.9 \%$ increase in performance with the conventional single coil ferrite rod antenna (SCFR). They claim to compare many surrounding energy sources and review their pros and cons.

Cao et al., (2019) Proposed an RF energy harvesting (RFEH) scheme that uses a tightly coupled electromagnetic resonance technology. A relay resonator placed in the middle of the tag antenna and the reader increases the PCE of the module. The relay resonator design considers the coupling concept of the resonant technique and near-field magnetic field.

Amrutha et al.,(2017).Proposed a Rectenna employing a microstrip patch with cement substrate that resonates at the RMS value of the AC voltage developed at antenna terminal when a plane wave is incident .The design is verified by simulations carried out with High Frequency Structure Simulator (HFSS). The results indicate the voltages are produced are in the range $0-5 \mathrm{mV}$.

Ghosh et al.,(2014). Proposed a RF energy harvesting system that is capable of powering the tiny wireless sensor nodes using the electromagnetic radiated energy from cell towers operating for CDMA and GSM bands. To harvest maximum energy from the radiating towers a special type of monopole antenna with microstrip is designed that has an impedance of $377 \Omega$ with circular polarization. The RF to DC conversion module uses a 7 stage voltage doubler circuit.

Ipar et al., (2016).Proposed an energy harvesting scheme using patch antenna made up micro strip that operates at 2.4GHz. The proposed RF energy harvesting technique is implemented for many electronic devices like LED, scientific calculator and battery charging. The RF method designed is reviewed using simulation and practical experiments.

Saini et al.,(2016).Proposed a power management scheme for RF energy harvesting. Design Features reveal that the maximum power efficiency is gained for specific range of load resistance. So the input resistance of DC-DC booster is same as the load resistance in order to get maximum power conversion. The circuit is fabricated using $180 \mathrm{~nm}$ mixed-mode CMOS technology.

Sim et al., (2009).Proposed a energy harvesting scheme using micro strip patch receiving antennatoharvestRFenergy for wireless soil sensor network implemented outdoor. The performance parameters evaluated are in terms of return loss, radiation efficiency, and gain. 


\subsection{RF Matching Circuits}

An impedance matching circuit is always found in between source and load, so as to transfer maximum power to the load while delivering an input impedance that is same as the complex conjugate of the source's output impedance.

Hammed et al., (2017).Proposed a methodology of a matching network selection strategy for variable input power levels to increase the harvested energy for a given input power levels. The RF energy harvester circuit uses an impedance circuitry that is off chip so as to increase the generated power with a specified frequency band of $902-$ 928 MHZ. The proposed RF energy harvester claims a PCE of $32 \%$ at $-15 \mathrm{dBm}(32 \mu \mathrm{W})$ and produces $3.2 \mathrm{~V}$ of DC voltage to a load of $1 \mathrm{M} \Omega$.

Felini et al.,(2014) Proposed an RF energy harvesting system with an improved dynamic impedance matching network (DyIMN). The DyIMN system was integrated on an FR4 substrate that uses the discrete components and is capable of converting RF energy to regulated DC voltage that can power many wireless devices. The experimental results claim to achieve an maximum impedance matching with an input $\mathrm{RF}$ power of upto $+5 \mathrm{dBm}$. The circuit requires as low as $-10 \mathrm{dBm}$ of power to harvest energy from a source with a distance of one $150 \mathrm{cms}$.

Hameed et al.,(2013).Proposed an RF-DC rectifier using a passive multi-stage circuit implemented on standard PMOS transistors to allow individual bulk biasing and thus avoiding the necessity of deep $\mathrm{n}$ well technology. The proposed 915-MHZ rectifier is fabricated with 130-nm CMOS technology. The design is capable of generating $1 \mathrm{~V}$ output with an received power of of $-22 \mathrm{dBm}$.

Khansalee et al.,(2015).Proposed a rectifier design with a source-pull simulation to estimate the maximum impedance matching to maximize the efficiency to $35.53 \%$ thereby producing an DC voltage $1.09 \mathrm{~V}$ and $3.16 \mathrm{~mA}$. The DC currents of $10 \mathrm{dBm}$ is produced at the load using $2420 \mathrm{MHz}$ continuous wave signal.

\subsection{Rectifiers}

A rectifier is an electrical device that converts one form of energy(AC) to the other form of energy (DC), which flows in only one direction.

Lee et al., (2018). Proposed adjustable impedance M/N with a CMOS technology to produce RF energy using $3 \mathrm{G} / 4 \mathrm{G}$ cellular low-band signals. The proposed converter consists of cross coupled rectifier with differential- drive and a four-bit capacitor array.The RF-to-DC rectification has a varying conversion efficiency with different loads.

Kim, et al., (2013). Proposed rectifier that uses multi-band impedance matching circuit with Villard structure, a lowpass filter (LPF) to suppress wideband harmonics and a load terminator. The circuit receives in band signals and cuts off the out-of-band signals so as to effectively block the back-scattered nonlinear frequency components from the nonlinear rectifying diodes to the antenna. The fabricated scheme results in high conversion efficiency .

Li et al., (2019) Proposed a rectifier circuit that utilizes the MOSFET threshold voltage for RF energy harvesting. The rectifier is designed with SMIC 0.18_m CMOS technology with an improved efficiency of $72.3 \%$.

$\mathrm{Wu}$ et al., (2016). Propose a simple converter that uses
940 MHZ signals for reduced-power wireless power transfer (WPT) system. By testing various diodes and topologies, a rectifier circuit is designed as a very-low ambient energy collecting system.

Inin Wang et al.,(2015) Proposed a design for RF energy harvesting using enhanced full-wave CMOS rectifying charge pump. They have used body-connected PMOS diodes for rectification and switching for capacitor charging pump with low-voltage operation of $0.4 \mathrm{~V}$.

Lin et al., (2017). Proposed a harvesting circuit that is designed using $0.18 \mu \mathrm{m}$ CMOS power-management system for wide range of ultra-high frequency (UHF). The architecture claims to be power-aware and has adaptive DC-DC conversion ratios with PCE 59\%.

Saffari et al., (2018). Proposed a four-stage threshold voltage compensated power converter to supply a increased power conversion efficiency (PCE) over a vast input power range that maximizes the area powered by wireless devices. The circuit is designed and verified using IBM 130-nm CMOS technology.

Alippi, et al., (2008). Proposed a low-power maximum power point tracker (MPPT) circuit particularly to powerup wireless sensor nodes and to store solar energy into rechargeable batteries even in unfavorable climatic conditions. The PCE is increased by using an modified ad hoc algorithm .

Saffari et al.,(2019) Proposed a RF converter scheme with four-stage threshold voltage compensation circuit that uses an sub threshold auxiliary transistors to achieve higher efficiency. The rectifier is designed in $130 \mathrm{~nm}$ CMOS technology.This technique can be applied to a link of considerably reduced number of rectifier stages.

Zhang et al,.(2014). Proposed an rectifier circuit to generate power from the surrounding energy sources with shunted Schottky diode to achieve maximum efficiency of $81 \%$. The PCE attained is around $50 \%$ of the input power level at $25 \mathrm{~m} \mathrm{~W}$ for a frequency of $1.43 \mathrm{GHz}$.

Mansourt et al., (2018).Proposed a exponential rectifier (DR) design for producing energy from radiofrequency with a power of $0 \mathrm{dBm}$. They propose to increase the efficiency over different frequency ranges with reduced size of RF rectifier circuit. The topology claims to provide an efficiency of more than $30 \%$. Also, the proposed topology claims the PCE more than $30 \%$ over a wide range of frequencies from 950 to $1500 \mathrm{MHz}$. Commercial balanced-unbalanced (BALUN) component is used for port transformation. The design claims to work for varying carrier frequencies with PCE of $57.5 \%$ at $1.1 \mathrm{GHz}$.

Iwata et al., (2016). Proposed an antenna with large impednce with Gate Control Diode optimised for RF energy harvesting. The rectification efficiency of $15 \%$ was achieved because of discarding the parasitic capacitance (Cj) of the diode with an input power of $-15 \mathrm{dBm}$ and frequency of 500MHZ.

Kim et al., (2019). Proposed an power generating scheme using CMOS inter coupled RF energy rectifier that increases energy efficiency by decreasing reverse saturation current. The rectifier is fabricated with $0.18 \mu \mathrm{m}$ CMOS technology for UHF band applications to gain a 
maximum PCE of $75 \%$ for $1 \mathrm{~V}$ output voltage. And also claim to have improved PCE by $8.8 \%$ than the existing rectifier circuits.

Zhang et al., (2017).Propose a exponentially-fed charge pumping rectifier design for generating power from surrounding RF energy sources with improved power efficiency. The simulation and measurements reveal that the efficiency of $5 \%$ is improved than the ultralow power rectifier with output DC voltage of $0.7 \mathrm{~V}$ amplitude.

Chaour et al.,(2017). Have proposed a rectifier design for Low power RF Energy Harvesting at $868 \mathrm{MHZ}$. The circuit is a passive RF-DC converter using a reactive matching circuit and voltage multiplier. They claim to harvest the RF energy at $40 \mathrm{dBm}$ of power for a resistive load of $50 \mathrm{~kW}$.

Sedeek et al.,(2018) .Proposed a $2.45 \mathrm{GHz}$ reduced power rectifier for generating power from radio frequency (RF) with $180^{\circ}$ hybrid junction. The proposed rectifier has two rectifying branches and two output ports. The circuit delivers maximum power efficiency of $70 \%$ than the usual voltage doubler circuit.

Mouapi et al.,(2017). Proposed a design for a small rectifying antenna (rectenna) that operates at GSM band to generate power from RF energy. The rectifier circuit is RF micro-generator miniaturized dipole antenna whose design is based on a Schenkel voltage multiplier circuit using Schottky diodes HSMS 2850 of AVAGO. Experimental results claim an PCE of $40 \%$.

Wang Y et al., (2014).Proposed a radio frequency harvesting scheme using gate cross-coupled (FGCC) rectifier structure to achieve better performance at LIP levels. The 3-stage FGCC rectifier uses small-thresholdvoltage (LTV) transistors so as to maximize the efficiency. For $850 \mathrm{MHZ}$ input AC signal, the PCE attained is $70 \%$ at $20 \mu \mathrm{W}(-17.0 \mathrm{dBm})$ input power .

\subsection{Voltage Doublers}

A voltage multiplier or an Voltage Doubler is an electrical circuit that increases the lower AC voltage to higher DC voltage, by using appropriate capacitors and diodes. Normally the Voltage multipliers find their application in electronic appliances to increase the required voltage levels , also used in high energy physics experiments and lightning safety testing.

Ullah et al., (2018). Proposed a small rectenna for RF energy harvesting, claim to improve the efficiency by using voltage doubling concept. The receiver antenna is a multiband monopole design implemented for the purpose of increased gain and uni-directional radiation pattern. At each frequency band special types of voltage multipliers are implemented to increase the output power. The design of antenna and a rectifier is carried on single FR-4 substrate to increase PCE to $85 \%$.

Chouhan et al., (2016) Proposed a voltage multiplier by using a simple cascading technique for $\mathrm{RF}$ energy harvesting. A $0.18 \mu \mathrm{m}$ CMOS technology is used to fabricate the proposed architecture. Resistors of $5 \mathrm{k}, 9 \mathrm{k}, 30 \mathrm{k}$ and $100 \mathrm{k}$ were used for measurement and to imitate practical load conditions. The power conversion efficiency (PCE) is the performance factor and it was done for input power levels from $20 \mathrm{dBm}$ to $0 \mathrm{dBm}$. The rectifier exhibited a maximum PCE of $74 \%$ at the input RF level of $2 \mathrm{dBm}$ for the load resistance of $5 \mathrm{k} \Omega$.

Umesao, Ryo, et al., (2014). Proposed rectifiers using the Gate Controlled Diodes(GCD) with the varying threshold voltage $(\mathrm{Vt})$ of the MOS by the ion-implantation, integrated with 0.18um CMOS technology. The DC values of the Gate Controlled Diodes claimed that the turn on voltage of the GCD is less than the voltage of Schottky Barrier Diode (SBD).

Jabbar et al.,(2010) Proposed an alternative version of existing C-MOS based voltage multiplier to attain PCE of $160 \%$ more than the existing designs with an input power of $0 \mathrm{dBm}$. RF energy harvesting circuit using Schottky diode was verified for both practical and simulations also.

\subsection{Hybrid Models}

Collado et al., (2013). Proposed an energy harvesting scheme by combining solar cell and a rectenna to collect surrounding energy. A $56 \mathrm{~mW}$ DC power is produced when the solar cell was illuminated with $100 \mathrm{w} / \mathrm{cm} 2$ solar irradiance and an power increase of $15 \%$ was recorded around $0.85 \mathrm{Ghz}$ and $1.85 \mathrm{Ghz}$ when illuminated by a microwave signal of $20 \mathrm{dBm}$.

Jadhav et al., (2017). Propose a design of hybrid circuit energy to harvest energy using both solar cell and antenna to tap the energy from surrounding energy resources. To harvest solar energy MPPT algorithm (P\&O Algorithm) is used with an antenna made up of Micro strip feed Patch operating at $2.4 \mathrm{GHz}$. The circuit reveals to produce a power of 9 Watts.

Niotaki,.et al(2014) have proposed EM energy harvesting scheme that incorporates solar and antenna structures as the source for energy harvesting circuits. This scheme uses "solar-to-electromagnetic" converters to supply the required power for RF signals generators. Also they have verified some of the new topologies to reduce the rectifier sensitivity to the received RF energy levels.

Nguyen,. et al (2018) Propose an hybrid energy harvester that uses RF energy and vibration energy. They describe the hybrid model that combines RF energy and thin piezoelectric film of polyvinylidene fluoride to harvest energy for frequency of $915 \mathrm{MHz}$ and at a vibration of $15 \mathrm{~Hz}$. They Have used a multistage voltage multiplier along with Dickson RF to DC converter. Results claim that a power of $3 \mathrm{w}$ and $1.8 \mu \mathrm{w}$ is generated by harvesting $\mathrm{RF}$ energy and vibration energy respectively

Yildiz et,.al (2018) Proposed an hybrid Energy harvesting scheme that uses solar and electromagnetic energies. They proposed a frame work known as MIP (mixed integer programming) to decrease energy consumed by the sensor nodes. The MIP is used as a reference to analyze the power consumed by the sensor nodes. The results claim to reduce the energy consumption by $90 \%$

\section{PRACTİCAL CHALLENGES}

- The efficiency of RFEH circuit decreases with the increase in the separation of the RF radiating source and the RF energy harvesting circuit.

- Requires a design of antenna with high gain using suitable materials and with required geometry.

- A suitable impedance matching network that maps 
to the impedance of the antenna and the rectifier and increase the efficiency of the network.

- The Radio Frequency DC power efficiency largely relies on the output power density of the converter.

- Difficulty in reducing the size of the antennas so as to match up for all kinds of electronic devices.

\section{CONCLUSIONS}

In this paper, we presented various protocols and techniques considered for the implementation of crosslayer design, RF components for WSN along RF energy harvesting techniques. The cross-layer design improves the performance by inclusion of layers interaction to gain extended energy efficiency, high reliability in packet delivery, and stability in multi-hop wireless sensor networks. RF energy signals are prevalent compared to the other ambient energy sources such as solar, thermal gradients and mechanical vibrations. In spite of the lower RF power density of the amount of energy generated is optimal to power up many wireless devices. Based on the findings in section 2 and 3 it reveals that there is lot of scope for hybrid models that consider more than one source of energy and a specific protocol for the TCP/IP layers under consideration for cross layer design. The study highlights the demand for an hybrid model that encompasses the energy efficient cross layered design along with an suitable RF energy harvesting system.

\section{REFERENCES}

[1] Choi JY, Kim HS, Baek I, Kwon WH. Cell based energy density aware routing: a new protocol for improving the lifetime of wireless sensor networks. Computer Communications 2005;28(11):1293-302.

[2] Chabalala, S. C., T. N. Muddenahalli, and F. Takawira. "Crosslayer adaptive routing protocol for wireless sensor networks." AFRICON, 2011. IEEE, 2011.

[3] Srivastava, Vineet, and Mehul Motani. "Cross-layer design: a survey and the road ahead." IEEE Communications magazine 43.12 (2005): 112-119

[4] Misra S, Reisslein M, Xue G. A survey of multimedia streaming in wireless sensor networks. IEEE Communications Surveys \& Tutorials 2008;10(4):18-39.

[5] Kulkarni S, Iyer A, Rosenberg C. An address-light, integrated mac and routing protocol for wireless sensor networks an addresslight, integrated MAC and routing protocol for wireless sensor networks. IEEE/ACM Transactions on Networking 2006;14(4):793-806.

[6] Choi JY, Kim HS, Baek I, Kwon WH. Cell based energy density aware routing: a new protocol for improving the lifetime of wireless sensor networks. Computer Communications 2005;28(11):1293-302.

[7] Yuan Y, Yang Z, He Z, He J. An integrated energy aware wireless transmission system for qos provisioning in wireless sensor network. Computer Communications 2006b;29(2):162-72.

[8] Shu T, Krunz M. Energy-efficient power/rate control and scheduling in hybrid TDMA/CDMA wireless sensor networks. Computer Networks 2009;53(9):1395-408.

[9] Kim, Sangkil, et al., "Ambient RF energy-harvesting technologies for self-sustainable standalone wireless sensor platforms." Proceedings of the IEEE 102.11 (2014): 1649-1666.

[10] Jaffe, Paul, and James McSpadden. "Energy conversion and transmission modules for space solar power." Proceedings of the IEEE 101.6 (2013): 1424-1437.

[11] Martin A. Green, Keith Emery, Yoshihiro Hishikawa, Wilhelm Wartaand Ewan D. Dunlop. Solar cell effciency tables (Version 38). Progress in Photovoltaics: Research and Applications,
19(5):565\{572, 2011.

[12] Tesla, N. The transmission of electric energy without wires. New York: Electrical World and Engineer, 1904, p.162. Google Scholar.

[13] Yildiz, Faruk. "Potential Ambient Energy-Harvesting Sources and Techniques." Journal of technology Studies 35.1 (2009): 40-48.

[14] Piñuela, Manuel, Paul D. Mitcheson, and Stepan Lucyszyn. "Ambient RF energy harvesting in urban and semi-urban environments." IEEE Transactions on microwave theory and techniques 61.7 (2013): 2715-2726.

[15] Andrenko, AS, Lin, X, Zeng, M. Outdoor RF spectral survey: a roadmap for ambient RF energy harvesting. In: Proceedings of the TENCON 2015 IEEE region 10 conference, Taipa, Macau, 14 November 2015, pp.1-4. New York: IEEE.

[16] Nasab, SH, Asefi, M, Albasha, L. Investigation of RF signal energy harvesting. Active Passive Electron Comp 2010; 35: 22 23. Google Scholar.

[17] Lu, X. Wireless networks with RF energy harvesting: a contemporary survey. IEEE Commun Survey Tutorial 2015 17: 757-789. Google Scholar | Crossref | ISI

[18] Mascareñas, D, Flynn, E, Farrar, C. A mobile host approach for wireless powering and interrogation of structural health monitoring sensor networks. IEEE Sens J 2010; 9: 1719-1726. Google Scholar | Crossref | ISI.

[19] Ammar, Amira Ben, et al., "Cross-Layer Approach Based Energy Minimization for Wireless Sensor Networks." Wireless Personal Communications 98.2 (2018): 2211-2221.

[20] Chen, Wei, et al., "Cross layer design of heterogeneous virtual MIMO radio networks with multi-optimization." Parallel \& Distributed Processing, Workshops and Phd Forum (IPDPSW), 2010 IEEE International Symposium on. IEEE, 2010.

[21] Di Marco, Piergiuseppe, et al., "TREnD: a timely, reliable, energy-efficient and dynamic wsn protocol for control applications." 2010 IEEE International Conference on Communications, ICC 2010; Cape Town. 2010

[22] Babulal, Kanojia Sindhuben, and Rajiv Ranjan Tewari. "Cross layer energy efficient routing (XLE2R) for prolonging lifetime of wireless sensor networks." Computer and Communication Technology (ICCCT), 2010 International Conference on. IEEE 2010 .

[23] Chen, Yongrui, Weidong Yi, and Yang Yang. "Cross-layer strategy for energy-efficient cooperative communication in wireless sensor networks." (2010): 203-208.

[24] Chabalala, S. C., T. N. Muddenahalli, and F. Takawira. "Crosslayer adaptive routing protocol for wireless sensor networks." AFRICON, 2011. IEEE, 2011.

[25] Dessales, Denis, et al., "Case Study of a Wireless Sensor Network for a Building Monitoring Application." Green Computing and Communications (GreenCom), 2012 IEEE International Conference on. IEEE, 2012.

[26] Gao, Fei, et al., "Design and optimization of a cross-layer routing protocol for multi-hop wireless sensor networks." Sensor Network Security Technology and Privacy Communication System (SNS \& PCS), 2013 International Conference on. IEEE 2013.

[27] Jaradat, Toleen, et al., "Energy efficient cross-layer routing protocol in wireless sensor networks based on fuzzy logic." Wireless Communications and Mobile Computing Conference (IWCMC), 2013 9th International. IEEE, 2013.

[28] Anupama, K. R., et al., "A wireless sensor network-based pipeline monitoring system." Signal Processing and Integrated Networks (SPIN), 2014 International Conference on. IEEE, 2014

[29] Chen, Yongrui, et al., "Cross-layer design for energy-efficient reliable routing in wireless sensor networks." Mobile Ad-hoc and Sensor Networks (MSN), 2015 11th International Conference on. IEEE, 2015.

[30] Alhalafi, Abdullah, et al., "gTBS: A green task-based sensing for energy efficient wireless sensor networks." Computer Communications Workshops (INFOCOM WKSHPS), 2016 IEEE Conference on. IEEE, 2016.

[31] Mishra, Mukesh, Gourab Sen Gupta, and Xiang Gui. "A review of and a proposal for cross-layer design for efficient routing and secure data aggregation over WSN." Computational Intelligence 
and Networks (CINE), 2017 3rd International Conference on. IEEE, 2017

[32] He, Shibo, et al., "Cross-layer optimization of correlated data gathering in wireless sensor networks." IEEE Transactions on Mobile Computing 11.11 (2012): 1678-1691.

[33] Zhang, Haiyang. "Classic efficient-energy MAC protocols for wireless sensor networks." Wireless Communications Networking and Mobile Computing (WiCOM), 2010 6th International Conference on. IEEE, 2010.

[34] Dessales, Denis, et al., "Physical layer study in a goal of robustness and energy efficiency for wireless sensor networks." Design and Architectures for Signal and Image Processing (DASIP), 2010 Conference on. IEEE, 2010.

[35] Shen, Jian, et al., "Energy efficiency of mac protocols in wireless sensor networks." Information Science and Applications (ICISA), 2011 International Conference on. IEEE, 2011

[36] Karvonen, Heikki, and Carlos Pomalaza-Ráez. "A cross layer design of coding and awake/sleep periods in WSNS." Personal, Indoor and Mobile Radio Communications, 2006 IEEE 17th International Symposium on. IEEE, 2006.

[37] Tiglao, Nestor Michael C., and Antonio M. Grilo. "Cross-layer caching based optimization for wireless multimedia sensor networks." Wireless and mobile computing, networking and communications (WiMob), 2012 IEEE 8th international conference on. IEEE, 2012.

[38] Hsueh, Ching-Tsung, Chih-Yu Wen, and Yen-Chieh Ouyang. "Two-tier receiver-initiated secure scheme for hierarchical wireless sensor networks." ITS Telecommunications (ITST), 2012 12th International Conference on. IEEE, 2012.

[39] Sadouq, Zouhair A., Marouane El Mabrouk, and Mohamed Essaaidi. "Conserving energy in WSN through clustering and power control." Information Science and Technology (CIST), 2014 Third IEEE International Colloquium in. IEEE, 2014

[40] Sadiq, Ali Safa, et al., "An Energy-Efficient Cross-Layer approach for cloud wireless green communications." Fog and Mobile Edge Computing (FMEC), 2017 Second International Conference on. IEEE, 2017

[41] Quintero, Vanessa, Claudio Estevez, and Marcos Orchard. "Stateof-charge estimation to improve energy conservation and extend battery life of wireless sensor network nodes." Ubiquitous and Future Networks (ICUFN), 2017 Ninth International Conference on. IEEE, 2017.

[42] Cho, Jaejoon, et al., "An energy-efficient mechanism using CLMAC protocol for wireless sensor networks." null. IEEE, 2007.

[43] Hsueh, Ching-Tsung, Chih-Yu Wen, and Yen-Chieh Ouyang. "Two-tier receiver-initiated secure scheme for hierarchical wireless sensor networks." ITS Telecommunications (ITST), 2012 12th International Conference on. IEEE, 2012.

[44] Bencini, Luca, et al., "A fault tolerant communication architecture supporting critical monitoring with Wireless Sensor Networks." MELECON 2010-2010 15th IEEE Mediterranean Electrotechnical Conference. IEEE, 2010.

[45] Song, Liang, and Dimitrios Hatzinakos. "A cross-layer architecture of wireless sensor networks for target tracking." IEEE/ACM Transactions on Networking (TON) 15.1 (2007): 145-158.

[46] Chen, Yongrui, Weidong Yi, and Yang Yang. "Cross-layer strategy for energy-efficient cooperative communication in wireless sensor networks." (2010): 203-208.

[47] (Xueying Zhang. "A cross-layer wireless sensor network energyefficient communication protocol for real-time monitoring of the long-distance electric transmission lines." Journal of Sensors 2015 (2015)

[48] Xiong, Binbin, et al., "Cross-layer optimal policies for stochastic reliable transmission in wireless sensor networks." Ultra Modern Telecommunications and Control Systems and Workshops (ICUMT), 2010 International Congress on. IEEE, 2010

[49] Cao, Shihua, and Jianqing Li. "A high efficiency twin coil ferrite rod antenna for RF energy harvesting in AM band." 2017 5th International Conference on Enterprise Systems (ES). IEEE, 2017.

[50] Cao, Xuan-Tu, and Wan-Young Chung. "An Enhanced Multiplication of RF Energy Harvesting Efficiency Using Relay Resonator for Food Monitoring." Sensors 19.9 (2019): 1963.
[51] Amrutha, M. V., and B. Sabarish Narayanan. "Cement based patch for low profile rectenna implementation for RF energy harvesting systems." 2017 International Conference on Technological Advancements in Power and Energy (TAP Energy). IEEE, 2017.

[52] Ghosh, Saswati, Soumya K. Ghosh, and Ajay Chakrabarty. "Design of RF energy harvesting system for wireless sensor node using circularly polarized monopole antenna: RF energy harvesting system for WSN node using circularly polarized antenna." 2014 9th international conference on industrial and information systems (ICIIS). IEEE, 2014.

[53] Ipar, Pankaj E., Shilpa M. Lambor, and Sangeeta M. Joshi. "Development of radio frequency energy harvesting module." 2016 IEEE Annual India Conference (INDICON) IEEE, 2016.

[54] Saini, Gaurav, et al., "Efficient power management circuit for RF energy harvesting with $74.27 \%$ efficiency at $623 \mathrm{nW}$ available power." 2016 14th IEEE International New Circuits and Systems Conference (NEWCAS). IEEE, 2016

[55] Sim, Zhi Wei, Roger Shuttleworth, and Bruce Grieve. "Investigation of PCB microstrip patch receiving antenna for outdoor RF energy harvesting in wireless sensor networks." 2009 Loughborough Antennas \& Propagation Conference. IEEE, 2009.

[56] Hameed, Zohaib, and Kambiz Moez. "Design of impedance matching circuits for RF energy harvesting systems." Microelectronics Journal 62 (2017): 49-56.

[57] Felini, C., M. Merenda, and F. G. Della Corte. "Dynamic impedance matching network for RF energy harvesting systems." 2014 IEEE RFID Technology and Applications Conference (RFID-TA). IEEE, 2014.

[58] Hameed, Zohaib, and Kambiz Moez. "Fully-integrated passive threshold-compensated PMOS rectifier for RF energy harvesting." 2013 IEEE 56th International Midwest Symposium on Circuits and Systems (MWSCAS). IEEE, 2013.

[59] Khansalee, Ekkaphol, Yan Zhao, and Kittipong Nuanyai. "High frequency rectifier for RF energy harvesting systems." 2015 7th International Conference on Information Technology and Electrical Engineering (ICITEE). IEEE, 2015

[60] Lee, Donggu, et al., "A CMOS Rectifier with 72.3\% RF-to-DC Conversion Efficiency Employing Tunable Impedance Matching Network for Ambient RF Energy Harvesting." 2018 International SoC Design Conference (ISOCC). IEEE, 2018.

[61] Kim, Phirun, Girdhari Chaudhary, and Yongchae Jeong. "A dualband RF energy harvesting using frequency limited dual-band impedance matching." Progress In Electromagnetics Research 141 (2013): 443-461.

[62] Li, Yan, et al., "A high efficiency CMOS RF rectifier for RF energy harvesting with dynamic self-body-biasing technique." IEICE Electronics Express (2019): 16-20190462.

[63] Wu, Xuyue, et al., "A high efficiency rectifier for ambient RF energy harvesting at $940 \mathrm{MHz} . " 2016$ IEEE International Conference on Microwave and Millimeter Wave Technology (ICMMT). Vol. 2. IEEE, 2016

[64] Wang, Weiyin, Hei Wong, and Yan Han. "A high-efficiency fullwave CMOS rectifying charge pump for RF energy harvesting applications." Microelectronics Journal 46.12 (2015): 1447-1452.

[65] Lin, Shu-Hsuan, et al., "A high-efficiency power management IC with power-aware multi-path rectifier for wide-range RF energy harvesting." 2017 IEEE MTT-S International Microwave Symposium (IMS). IEEE, 2017.

[66] Saffari, Parvaneh, Ali Basaligheh, and Kambiz Moez. "A WideRange Highly Power Efficient RF-to-DC Rectifier for RF Energy Harvesting Systems." 2018 IEEE International Symposium on Circuits and Systems (ISCAS). IEEE, 2018

[67] Alippi, Cesare, and Cristian Galperti. "An adaptive system for optimal solar energy harvesting in wireless sensor network nodes." IEEE Transactions on Circuits and Systems I: Regular Papers 55.6 (2008): 1742-1750.

[68] Saffari, Parvaneh, Ali Basaligheh, and Kambiz Moez. "An RF-toDC Rectifier With High Efficiency Over Wide Input Power Range for RF Energy Harvesting Applications." IEEE Transactions on Circuits and Systems I: Regular Papers (2019).

[69] Zhang, Hongxian, and Xinen Zhu. "A broadband high efficiency rectifier for ambient RF energy harvesting." 2014 IEEE MTT-S international microwave symposium (IMS2014). IEEE, 2014. 
[70] Mansour, Mohamed M., Xavier Le Polozec, and Haruichi Kanaya. "Compact and wide-band efficiency improved RF differential rectifier for wireless energy harvesting." 2018 IEEE/MTT-S International Microwave Symposium-IMS. IEEE, 2018.

[71] Iwata, Junpei, et al., "Confirmation of high efficiency on rectenna with high impedance antenna and optimized gate controlled diode for RF energy harvesting." 2016 IEEE SENSORS. IEEE, 2016.

[72] Kim, Hyeonwoo, and Ickjin Kwon. "Design of high-efficiency CMOS rectifier with low reverse leakage for RF energy harvesting." Electronics Letters 55.8 (2019): 446-448.

[73] Zhang, Hao, et al., "Differentially-fed charge pumping rectifier design with an enhanced efficiency for ambient RF energy harvesting." 2017 IEEE MTT-S International Microwave Symposium (IMS). IEEE, 2017.

[74] Chaour, Issam, Ahmed Fakhfakh, and Olfa Kanoun. "Enhanced passive RF-DC converter circuit efficiency for low RF energy harvesting." Sensors 17.3 (2017): 546

[75] Sedeek, Asmaa, Emad Tammam, and El-Sayed Hasaneen. "High Efficiency $2.45 \mathrm{GHz}$ Low Power Hybrid Junction Rectifier for RF Energy Harvesting." 2018 International Japan-Africa Conference on Electronics, Communications and Computations (JAC-ECC). IEEE, 2018.

[76] Mouapi, Alex, Nadir Hakem, and Nahi Kandil. "High efficiency rectifier for RF energy harvesting in the GSM band." 2017 IEEE International Symposium on Antennas and Propagation \& USNC/URSI National Radio Science Meeting. IEEE, 2017.

[77] Wang, Y., and M. Sawan. "High-efficiency CMOS rectifier dedicated for multi-band ambient RF energy harvesting." 2014 21st IEEE International Conference on Electronics, Circuits and Systems (ICECS). IEEE, 2014.

[78] Ullah, Shahid, et al., "Design of High Efficiency Multiband Rectenna for RF Energy Harvesting." 2018 Progress in Electromagnetics Research Symposium (PIERS-Toyama). IEEE, 2018.

[79] Chouhan, Shailesh Singh, Marko Nurmi, and Kari Halonen. "Efficiency enhanced voltage multiplier circuit for RF energy harvesting." Microelectronics journal 48 (2016): 95-102.

[80] Umesao, Ryo, et al., "High efficiency RF energy harvesting with threshold-votlage-adjusted gate control diode." 2014 IEEE Faible Tension Faible Consommation. IEEE, 2014.

[81] Jabbar, Hamid, Young S. Song, and Taikyeong Ted Jeong. "RF energy harvesting system and circuits for charging of mobile devices." IEEE Transactions on Consumer Electronics 56.1 (2010): 247-253.

[82] Collado, Ana, and Apostolos Georgiadis. "Conformal hybrid solar and electromagnetic (EM) energy harvesting rectenna." IEEE Transactions on Circuits and Systems I: Regular Papers 60.8 (2013): 2225-2234.

[83] Jadhav, Shruti B., and Shilpa M. Lambor. "Hybrid solar and radio frequency (RF) energy harvesting." 2017 IEEE International Conference on Power, Control, Signals and Instrumentation Engineering (ICPCSI). IEEE, 2017.

[84] Niotaki, Kyriaki, et al., "Solar/electromagnetic energy harvesting and wireless power transmission." Proceedings of the IEEE 102.11 (2014): 1712-1722.

[85] Nguyen, Son, and Rajeevan Amirtharajah. "A hybrid RF and vibration energy harvester for wearable devices." 2018 IEEE Applied Power Electronics Conference and Exposition (APEC). IEEE, 2018.

[86] Yildiz, Huseyin Ugur, Vehbi Cagri Gungor, and Bulent Tavli. "A hybrid energy harvesting framework for energy efficiency in wireless sensor networks based smart grid applications." 2018 17th Annual Mediterranean Ad Hoc Networking Workshop (Med-Hoc-Net). IEEE, 2018

[87] Saunders, S.; Aragón-Zavala, A. Antennas and Propagation for Wireless Communication Systems; John Wiley \& Sons: Hoboken, NJ, USA, 2007. [Google Scholar 